\title{
Effect of Alignment on Transport Properties of Carbon Nanotube/Metallic Junctions
}

\author{
Buzz Wincheski, Min Namkung, ${ }^{1}$ Jan Smits, ${ }^{2}$ Phillip Williams, and ${ }^{3}$ Robert Harvey \\ NASA LaRC \\ Hampton VA 23681 \\ ${ }^{1}$ Lockheed Martin Space Operation \\ Hampton VA, 23681 \\ ${ }^{2}$ National Research Council \\ Hampton VA, 23681 \\ ${ }^{3}$ Christopher Newport University \\ Newport News VA, 23606
}

\section{ABSTRACT}

Ballistic and spin coherent transport in single walled carbon nanotubes (SWCNT) are predicted to enable high sensitivity single-nanotube devices for strain and magnetic field sensing. Based upon these phenomena, electron beam lithography procedures have been developed to study the transport properties of purified HiPCO single walled carbon nanotubes for development into sensory materials for nondestructive evaluation. Purified nanotubes are dispersed in solvent suspension and then deposited on the device substrate before metallic contacts are defined and deposited through electron beam lithography. This procedure produces randomly dispersed ropes, typically $2-20 \mathrm{~nm}$ in diameter, of single walled carbon nanotubes. Transport and scanning probe microscopy studies have shown a good correlation between the junction resistance and tube density, alignment, and contact quality. In order to improve transport properties of the junctions a technique has been developed to align and concentrate nanotubes at specific locations on the substrate surface. Lithographic techniques are used to define local areas where high frequency electric fields are to be concentrated. Application of the fields while the substrate is exposed to nanotube-containing solution results in nanotube arrays aligned with the electric field lines. A second electron beam lithography layer is then used to deposit metallic contacts across the aligned tubes. Experimental measurements are presented showing the increased tube alignment and improvement in the transport properties of the junctions.

\section{INTRODUCTION}

Carbon nanotube based materials have been heavily researched lately due to the promising electronic properties, high strength, and low density of carbon nanotubes [1]. Multifunctional materials capitalizing on these attributes have the potential to enable the development of high strength, lightweight, materials with embedded sensing capabilities. In particular, NASA LaRC is studying single wall nanotube based materials for structural health monitoring applications including strain sensing and electromagnetic nondestructive evaluation [2]. In order to fabricate such materials systems, a significant effort has been placed on carbon nanotube dispersion, alignment, and nanotube/metallic junction quality. In this work, a method for directed placement of solution dispersed single wall carbon nanotubes across a surface is described. Nanotube alignment in localized positions across a $2 \mathrm{~mm}^{2}$ area has been 
demonstrated, and transport and scanning probe microscopy measurements show improved junction quality over randomly aligned systems.

\section{ELECTRIC FIELD INDUCED ALIGNMENT OF SINGLE WALLED CARBON NANOTUBES}

Recent reports by several authors have indicated the ability to position and align carbon nanotubes with high strength electric fields [3-8]. In these studies electric fields have been used to align single-walled carbon nanotubes during CVD growth [3] as well as to position both multi-wall [4-5] and single wall solvent dispersed tubes [6-8]. Experimental results have shown that AC and DC electric fields can be used to place and align CNTs in specific locations, while AC fields produce more reliable results with fewer contamination particles localized with the CNTs [5,7-8]. As it can be critical to localize and align CNTs in specific locations of a material system in order to achieve desired electronic and structural properties, these studies have provided a significant advance toward the incorporation of solution dispersed SWCNTs into novel multifunctional materials.

Following the studies discussed above, we have used ultraviolet and electron beam lithography procedures to produce samples in which multiple areas of high strength electric fields could be localized. Toluene dispersed SWCNTs were exposed to the samples while a 5 $\mathrm{MHz}$ field was applied between the multiple patterned electrodes. Results were analyzed through transport and scanning probe microscopy studies before a second electron beam lithography layer was used to deposit electrodes across the pre-aligned tubes. This method enables the positioning and alignment of CNTs in multiple predefined locations across a surface and then maximizes the CNT junction quality by depositing the critical junctions directly on top of the pre-positioned and aligned tubes.

The samples used in this study were prepared through UV and electron beam lithography as discussed in previous work [6]. A 100-mesh TEM grid is used as a UV mask to produce bonding pads and enable alignment of the electron beam system to the corners of the $205 \square \mathrm{m}$ x $205 \square \mathrm{m} \mathrm{Cr} / \mathrm{Au}$ pads. Electron beam lithography is then used to create the fine features of the pattern. In this work, an array of 12 electrodes was defined, in parallel, across the sample. Both $\mathrm{T}$ and point type electrodes have been fabricated. The pattern was laid out such that a second electron beam lithography could be performed after tube deposition to create twelve individual metal/CNT/metal junctions with the metal deposited directly over the pre-positioned and aligned tubes. Figure 1 displays an optical micrograph of a section of 3 junctions across a sample and a close up view of a single $T$ type junction. In the figure the junctions run from the upper left to the lower right corner. $1 \square \mathrm{m}$ wide lines have been deposited through electron beam lithography to connect the outer pads, define the T-junctions for trapping and aligning the CNTs, and to place the alignment marks for coordination with the second electron beam layer. This second layer places the $200 \mathrm{~nm}$ wide conduction electrodes between the alignment electrodes, on top of the pre-positioned and aligned tubes.

Carbon nanotube deposition was performed using purified SWCNTs purchased from Carbon Nanotechnologies, Inc. As discussed in previous work, a small amount of the asreceived tubes were dispersed in electronic grade toluene through ultrasonic agitation for 24 hours [6]. Following the UV and first electron beam lithography depositions, the sample was mounted under a video microscope and spring loaded contacts were positioned on the inner and outer contact pads. A $5 \mathrm{~V}_{\mathrm{rms}}$ sine wave at $5 \mathrm{MHz}$ was then applied to the pads to create an 

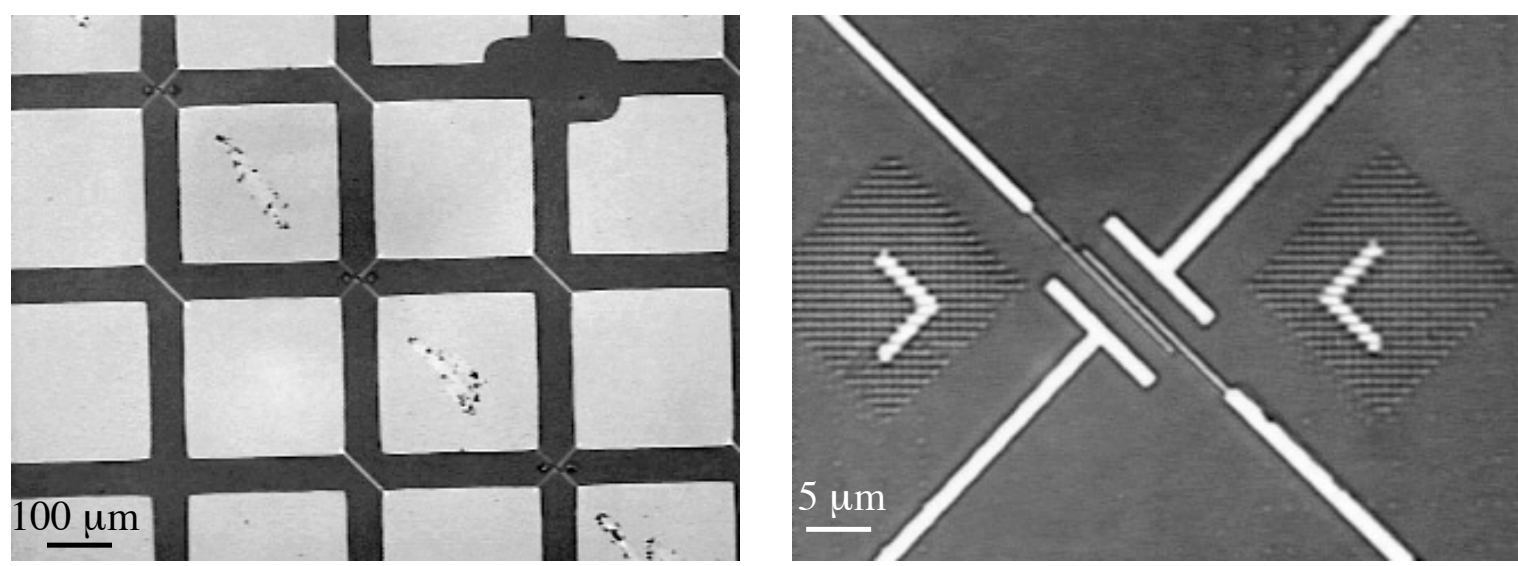

Figure 1. Optical micrograph of CNT alignment sample. Left figure shows section of 3 junctions from upper left to lower right hand corner. Square grid in the micrograph deposited through UV lithography using 100 mesh TEM grid mask. Finer features deposited through electron beam lithography. Right figure displays close up view of single junction.

electric field of approximately $1 \times 10^{6} \mathrm{~V}_{\mathrm{rms}} \mathrm{m}^{-1}$ between each of the twelve alignment electrodes. Approximately $20 \square \mathrm{L}$ of the toluene dispersed CNT suspension, enough to completely cover the sample surface, was deposited on the sample surface for 45 seconds. The sample was then blown dry and the electric field removed.

The effectiveness of the deposition was investigated by measuring the resistance of the twelve parallel alignment junctions. Typically, a resistance of 1 to $10 \mathrm{M} \square$ was found across the twelve parallel $5 \square \mathrm{m}$ gaps (the spacing between junctions was reduced to $3 \square \mathrm{m}$ for the point type electrodes). In some cases, resistance values in excess of $100 \mathrm{M} \square$ were measured. In these cases the deposition and alignment procedure described above was reapplied. At this stage the tube alignment was determined using scanning probe microscopy.

Figure 2 displays typical results for tube deposition and alignment between both the $\mathrm{T}$ and point type alignment electrodes. The scanning probe microscopy images were acquired with
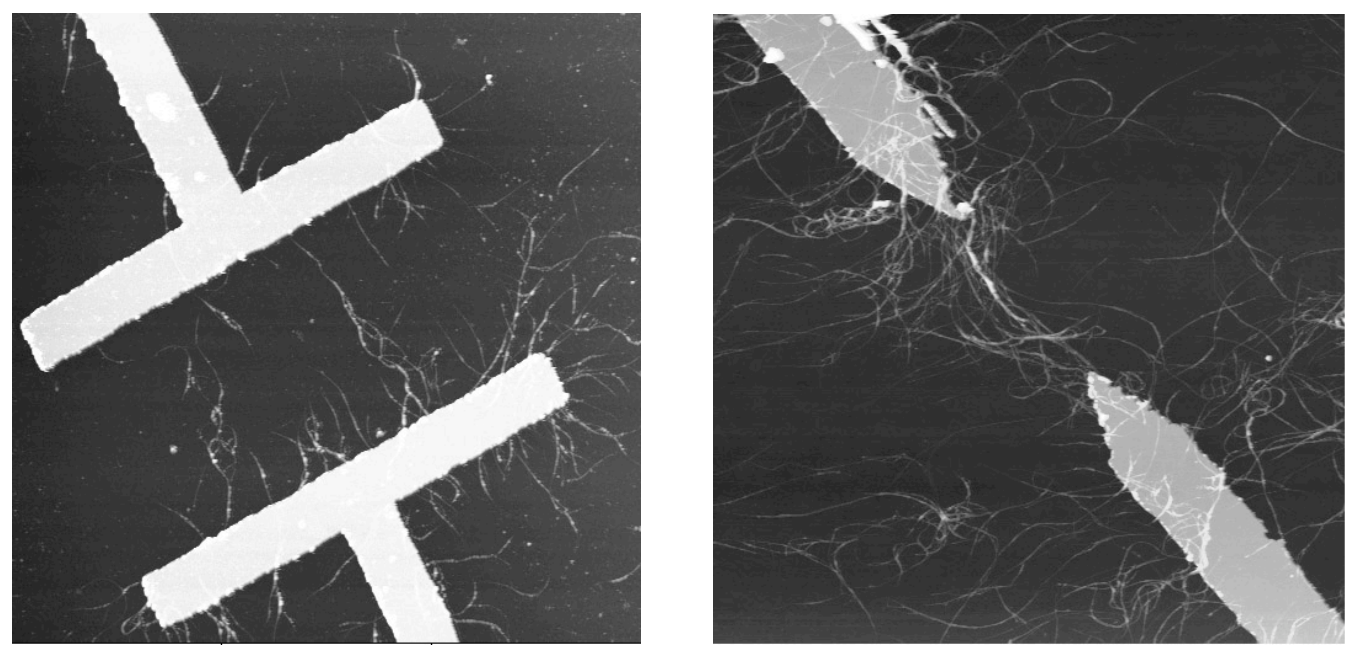

Figure 2. Carbon nanotube positioning and alignment. Left figure shows an AFM image of SWCNT ropes aligned by $\mathrm{T}$ type alignment electrodes. Right figure show results for point type electrodes. The spacing between the electrodes is $5 \square \mathrm{m}$ for the $\mathrm{T}$ and $3 \square \mathrm{m}$ for the point type 
a Digital Instruments Nanoscope IIIa Multimode microscopy operating in tapping mode. As can be seen in the figures, CNTs are concentrated in the areas of high electric field, following the electric field lines. For comparison, a finite element model of the electric field near a point type electrode is displayed in Figure 3. The model displays both the electric potential and electric field vectors for a $10 \mathrm{~V}$ dc bias between the two alignment electrodes. Symmetry considerations have been applied along the mid-plane between the electrodes to reduce the problem space. It is interesting to note that the experimental data shows tubes oriented perpendicular to the alignment electrodes, close to the conductors, in locations away from the electrode gap. Although this could be expected based upon the calculated electric field configuration, it was not noted in the work of Nagahara et al. using SWCNTs dispersed in Triton X-100 and aligned with a $5 \mathrm{MHz}$ electric field. In that work the reported tube trapping was isolated to locations directly between the electrodes [7].

Following the alignment of the SWCNTs, a second electron beam lithography layer was used to deposit conduction electrodes on top of the tubes. The lithography was performed relative to alignment marks deposited in the first electron beam lithography layer. In this manner the positioning of the conduction electrodes between the gaps of the alignment electrodes could be ensured. Figure 4 displays a scanning probe microscopy image of two completed junctions. The $\mathrm{T}$ type junction in figure 4 is the completed version of the corresponding junction of figure 2. The conduction electrodes are positioned directly between the alignment electrodes and on top of the CNTs aligned along the electric field lines in the gap.

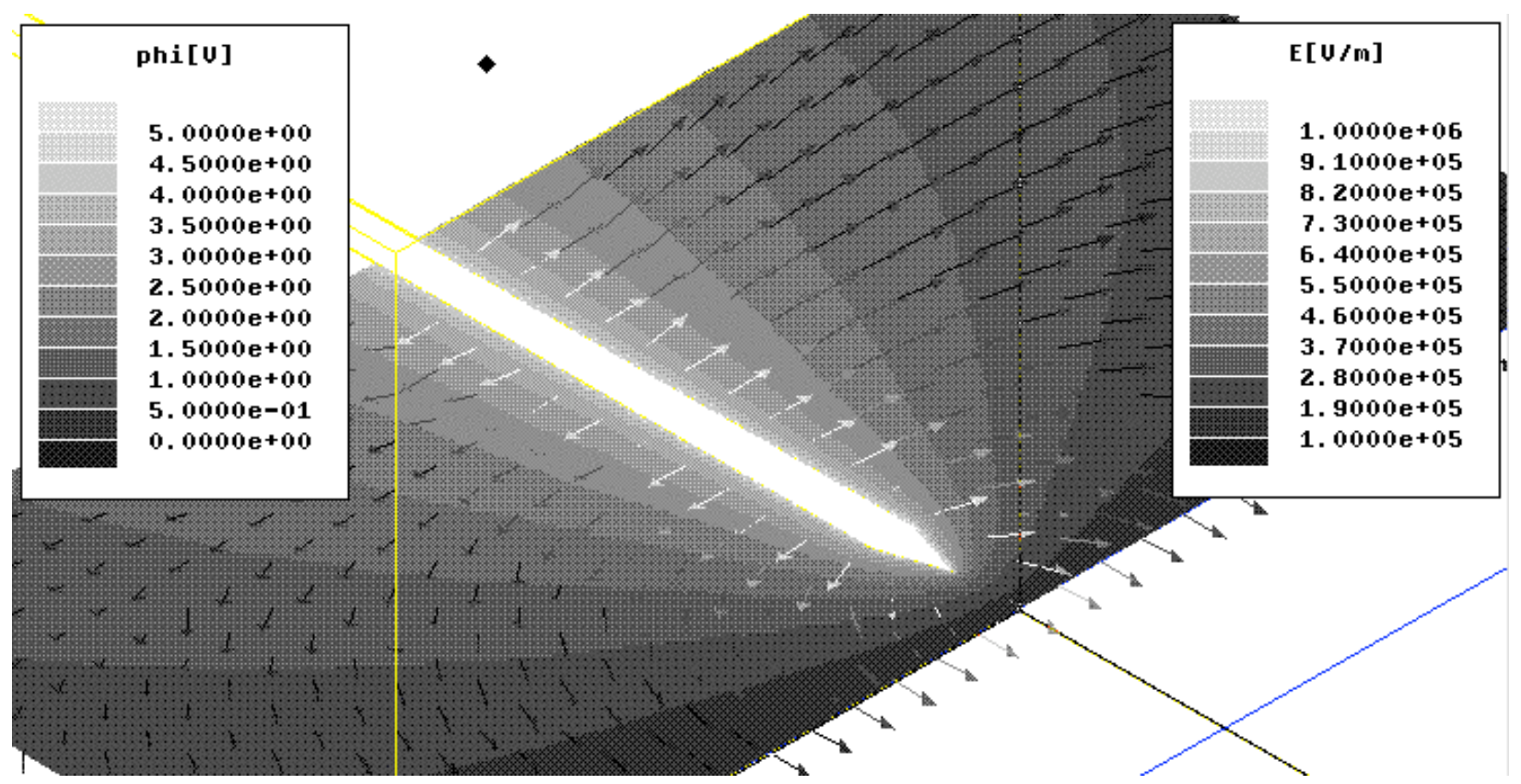

Figure 3. Finite element modeling results for electric potential and electric field vectors near point type alignment electrode. 

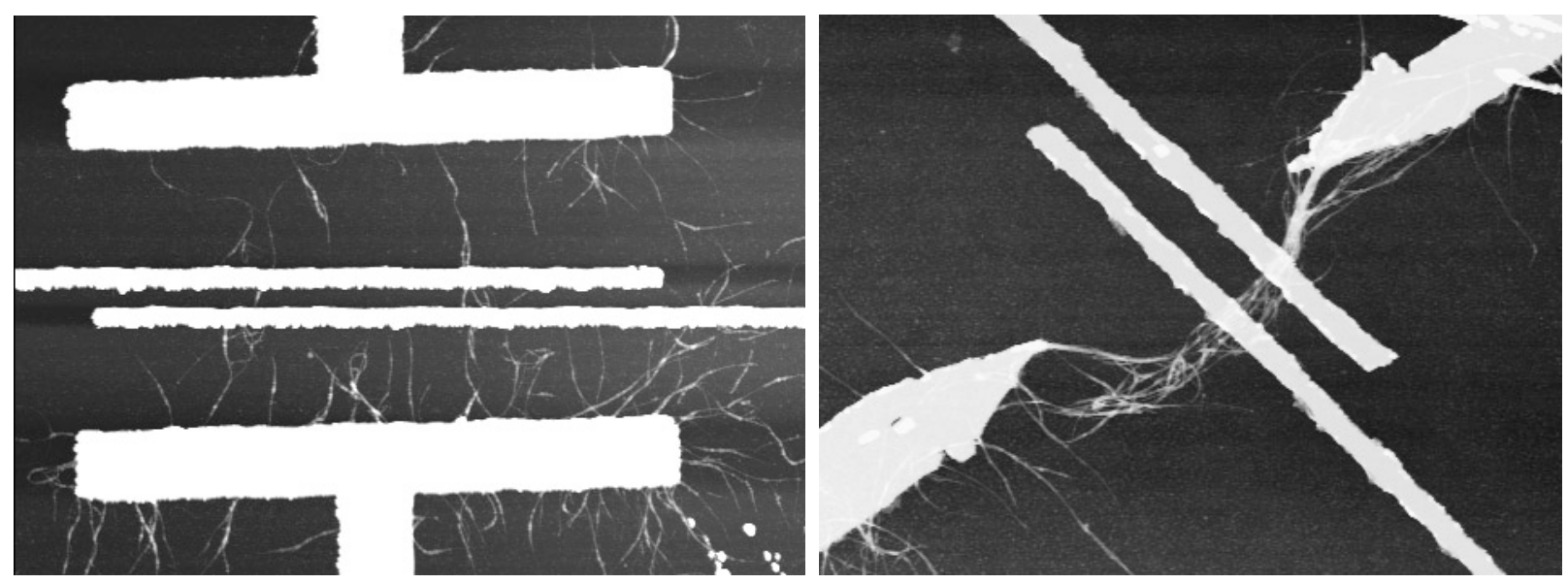

Figure 4. Final junctions showing conduction electrodes deposited on top of pre-positioned and aligned SWCNT ropes. Electrode spacing is $5 \square \mathrm{m}$ for the T and $3 \square \mathrm{m}$ for the point type electrodes.

\section{TRANSPORT MEASUREMENTS}

Electronic measurements of the CNT junctions were performed at room temperature using a two-point probe technique. The samples were mounted under a video microscope and spring-loaded point contacts were positioned on the bonding pads connected to the conduction electrodes of the junction. A Keithley 220 Programmable Current Source was used to supply a constant current through the junction while the voltage drop was measured with a Keithley 182 Sensitive Digital Voltmeter. The current-voltage measurements displayed in figure 5 were acquired under computer control. The results from four separate junctions are displayed in figure. The lowest resistance of approximately $10.6 \mathrm{k} \square$ is measured for junction 'Point 1'. This junction is pictured on the right hand side of figure 2. Conduction measurements were performed after the final electron beam layer deposition, not pictured here for brevity. The junctions labeled 'Point 2' and 'T Type' correspond to the point and T-junctions pictured in Figure 4. The final curve, labeled 'Unaligned', displays results for an unaligned random deposition of tubes with tube density and electrode spacing roughly equivalent to those pictured here [2]. The relatively high resistance values measured in this previous study initiated this research effort with the goal of reducing the contact resistance of solution dispersed CNT to metal junctions. The data are clearly encouraging. While typical junction resistances from our previous, randomly aligned studies were on the order of $100 \mathrm{k} \square$ to $10 \mathrm{M} \square$, values on the order of $10 \mathrm{k} \square$ for multiple junctions across the sample surface have now been routinely achieved.

\section{SUMMARY}

The application of electric fields during deposition of solution dispersed single walled carbon nanotubes has been shown to localize and align CNTs. A $5 \mathrm{MHz}, 5 \mathrm{~V}_{\text {rms }}$ electric field is used to concentrate tube deposition in locations of high electric field strength and align tube axes with the field vectors. By using a three-layer lithography procedure, alignment electrodes are fabricated over a large area of the material surface and, following tube deposition, conduction electrodes are placed over the positioned and aligned tubes. The procedure has been shown to 


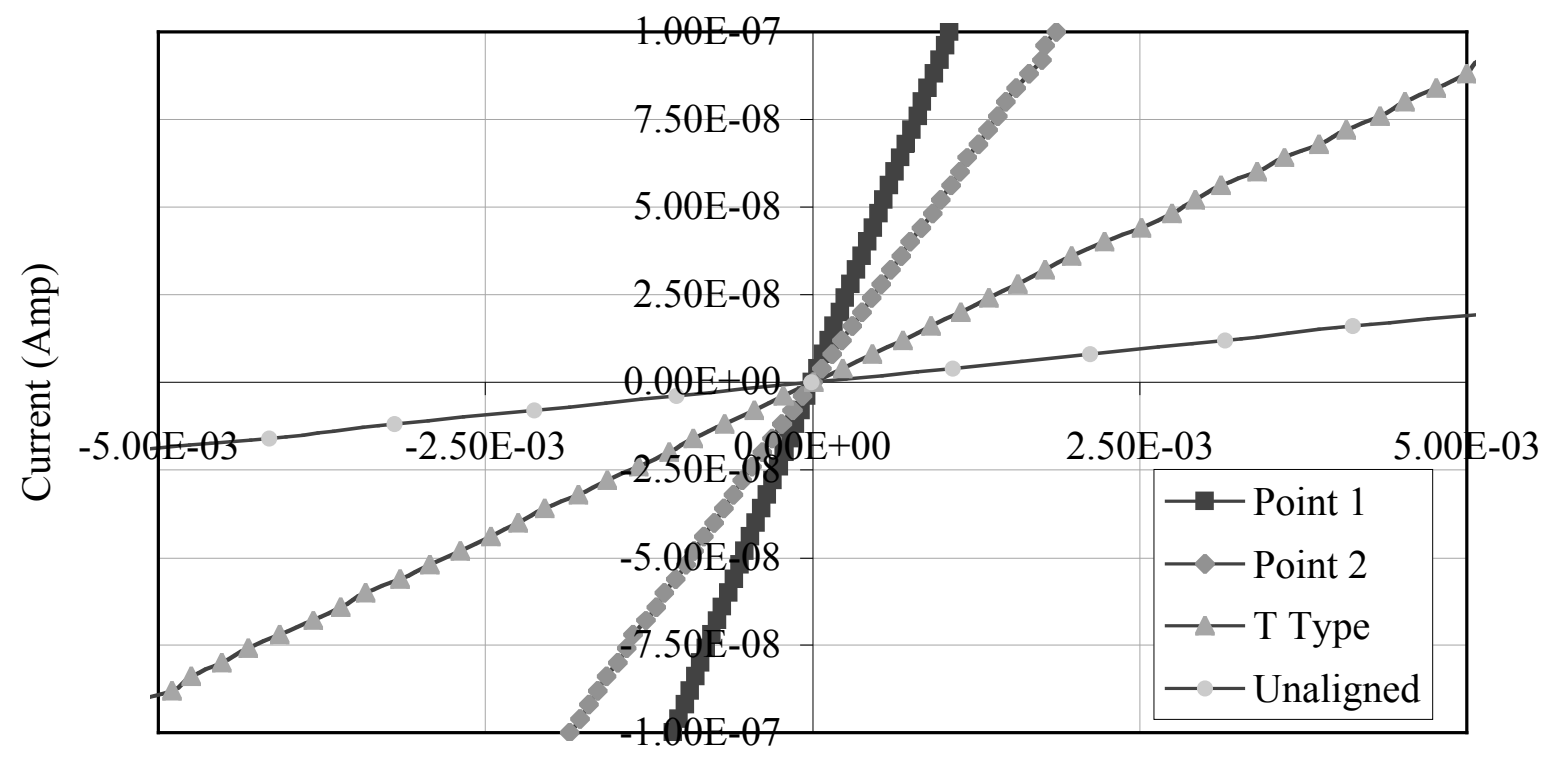

Voltage (V)

Figure 5. Current-voltage measurements for single walled carbon nanotube junctions with point type, $\mathrm{T}$ type, and unaligned tube positioning.

localize and align tubes in the desired locations and enable placement of conduction electrodes on top of the aligned tubes. Atomic force microscopy and conductivity measurements of the resulting metal/CNT/metal junctions show improved quality over randomly aligned systems. The results provide a mechanism for placement and alignment of tubes in critical locations of a material system. Current efforts are focused on utilizing this technique for the fabrication of multifunctional materials based upon solvent dispersed single wall carbon nanotubes.

\section{REFERENCES}

1. Carbon Nanotubes: Synthesis, Structure, Properties and Applications, edited by M. Dresselhaus, G. Dresselhaus, P. Avouris, Springer-Verlag, Berlin (2001).

2. B. Wincheski, M. Namkung, S.M. Paik, J. Smits, Mat Res. Soc. Symp. Proc., 721, E6.10.1 (2002).

3. A. Ural, Y. Li, and H. Dai, Applied Physics Letters, 18, 3464 (2002).

4. F. Wakaya, T. Nagai, K. Gamo, Microelectronic Engineering, 63, 27 (2002).

5. K. Yamamoto, S. Akita, and Y. Nakayama, J. Phys, D: Appl Phys., 31, L34 (1998).

6. J. Smits, B. Wincheski, J. Ingram, N. Watkins, and J. Jordan, Mat Res. Soc. Symp. Proc., 739, H7.11.1 (2003).

7. L. Nagahara, I. Amlani, J. Lewenstein, and R. Tsui, Applied Physics Letters, 80, 3826 (2002).

8. R. Krupke, F. Hennrich, H.B. Weber, D. Bechmann, O. Hampe, S. Malik, M.M. Kappes, H.V. Lohneysen, Applied Physics A - Materials Science and Processing, 76, 397 (2003). 